\title{
977-nm All-Fiber DFB Laser
}

\author{
L. B. Fu, M. Ibsen, D. J. Richardson, and D. N. Payne
}

\begin{abstract}
Three-level operation of a fiber distributed feedback (DFB) laser operating around $980 \mathrm{~nm}$ is demonstrated for the first time. The ytterbium-doped DFB laser shows a maximum slope efficiency of $61 \%$ and output power of $38.2 \mathrm{~mW}$ under a launched single-mode pump power of $150 \mathrm{~mW}$ at $910 \mathrm{~nm}$. The efficiency of lasers with different coupling strengths and at different pump wavelengths is also investigated.
\end{abstract}

Index Terms-Bragg gratings, distributed feedback (DFB) laser, optical fiber laser, ytterbium (Yb).

\section{INTRODUCTION}

$\mathbf{F}$ IBER distributed feedback (DFB) lasers are emerging as most promising candidates for dense wavelength-division multiplexing (WDM), LIDAR, fiber sensing, and spectroscopy. This is due to the fact that they possess a range of very desirable features such as inherent fiber compatibility, truly single-mode operation, very narrow linewidth (approximate kilohertz), low temperature sensitivity, nearly perfect performance in terms of ultralow relative intensity noise, high signal-to-noise ratio (SNR), and easy fabrication at predefined wavelengths. Furthermore, they can offer tens of nanometer tunability without requiring complex control mechanisms to maintain single-frequency operation [1].

It is of great interest to extend fiber DFB lasers to operate within new wavelength bands for a number of reasons, because they provide high-quality all-in fiber seeding sources for highpower amplifiers, and also can be served as narrow linewidth, high SNR sources for spectroscopy applications. Furthermore, there are applications in, for example, LIDAR which typically operate $\sim 1.9-2.1 \mu \mathrm{m}$. Previously, rare-earth doped fiber DFB lasers have been demonstrated to operate at $C$-band [2]-[4], $L$-band [5], [6], and $\sim 1 \mu \mathrm{m}$ [7]. However, it has long been recognized that it is difficult to achieve an efficient ytterbium (Yb)-doped fiber laser operating on the three-level transition in the wavelength range of $970-980 \mathrm{~nm}$. This has mainly been due to the strong absorption peak located around $977 \mathrm{~nm}$ [8] and strong competition from the quasi-four-level transition in the wavelength range of 1000-1200 nm. It should be noted that this is also the case for both cladding-pumped and core-pumped 980-nm fiber lasers.

In this letter, we experimentally demonstrate for the first time, to our knowledge, the operation of an all-fiber DFB laser operating at $977 \mathrm{~nm}$. With a launched pump power of $150 \mathrm{~mW}$ from

Manuscript received May 11, 2004; revised July 14, 2004. The work of M. Ibsen was supported by the Royal Society of London under a University Research Fellowship.

L. B. Fu is with CUDOS, University of Sydney, Sydney NSW 2006, Australia. M. Ibsen, D. J. Richardson, and D. N. Payne are with the Optoelectronics Research Centre, University of Southampton, Southampton SO17 1BJ, U.K. (e-mail: mi@orc.soton.ac.uk).

Digital Object Identifier 10.1109/LPT.2004.835619

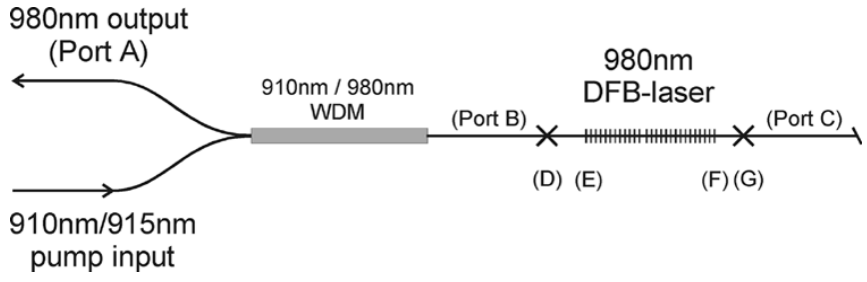

Fig. 1. Experimental setup (D, G: splicing points). Distance between splicing points: $|\mathrm{DG}| \approx 52 \mathrm{~mm}$. DFB grating length: $|\mathrm{EF}|=50 \mathrm{~mm}$.

a single-mode pump diode operating at $910 \mathrm{~nm}$, a fiber DFB laser with a designed coupling strength of $\kappa \sim 180 \mathrm{~m}^{-1}$ generates a total output power of $38.2 \mathrm{~mW}$. This laser exhibits a maximum slope efficiency of $61 \%$ although it is to be appreciated that the threshold is relatively high $(\sim 60 \mathrm{~mW})$ compared to fiber DFB lasers operating on more familiar transitions. We also report on our efforts to investigate the slope efficiencies of the 910-nm-pumped DFB lasers with slightly different coupling strengths, and the performance of a laser of fixed coupling strength but at the slightly different pump wavelengths of $915 \mathrm{~nm}$.

\section{Device Description AND EXPERIMENTAL SETUP}

The fiber used in these demonstrations was a phospho-silicate glass-host-based one codoped with concentrations of 1000-ppm Er and 20000-ppm Yb ions within the core. To render the fiber photosensitive enough for inscribing Bragg gratings using 244-nm continuous-wave (CW) ultraviolet (UV) light, it also had a B-Ge-Si doped annular ring to the core [9]. The small-signal absorption of the fiber was $110 \mathrm{~dB} / \mathrm{m}$ at $910 \mathrm{~nm}$ and $165 \mathrm{~dB} / \mathrm{m}$ at $915 \mathrm{~nm}$, which enables high efficiency operation for short length fiber lasers pumped at these wavelengths. Written into the fiber using $~ 100-\mathrm{mW} \mathrm{CW} \mathrm{UV-light}$ at $244 \mathrm{~nm}$ were 5 -cm-long $\pi$-phase-shifted DFB gratings of varying coupling strengths. To enable unidirectional operation, the phase shift was offset from the center of the DFB-grating during the writing process [3]. The fiber DFB lasers were subsequently annealed at $100^{\circ} \mathrm{C}$ for $12 \mathrm{~h}$ to stabilize the index modulations and then spliced to single-mode fibers at $980 \mathrm{~nm}$ (Points D and G in Fig. 1).

Fig. 1 illustrates the backward pump scheme used in our experiments. The 910-nm single-mode pump is launched into the common port (Port B) of a 910/980-nm WDM coupler which was spliced to the fiber DFB laser such that the desired 980-nm output emerges in the backward direction at Port A. The splice losses at Point $\mathrm{D}$ and $\mathrm{G}$ are both about $0.3 \mathrm{~dB}$ and the insertion loss of the WDM coupler at $980 \mathrm{~nm}$ is around $0.6 \mathrm{~dB}$. Note that the use of a 910-nm rather than a 915-nm single-mode pump diode in these experiments was merely down to commercial availability at the time of these experiments—similar results but 


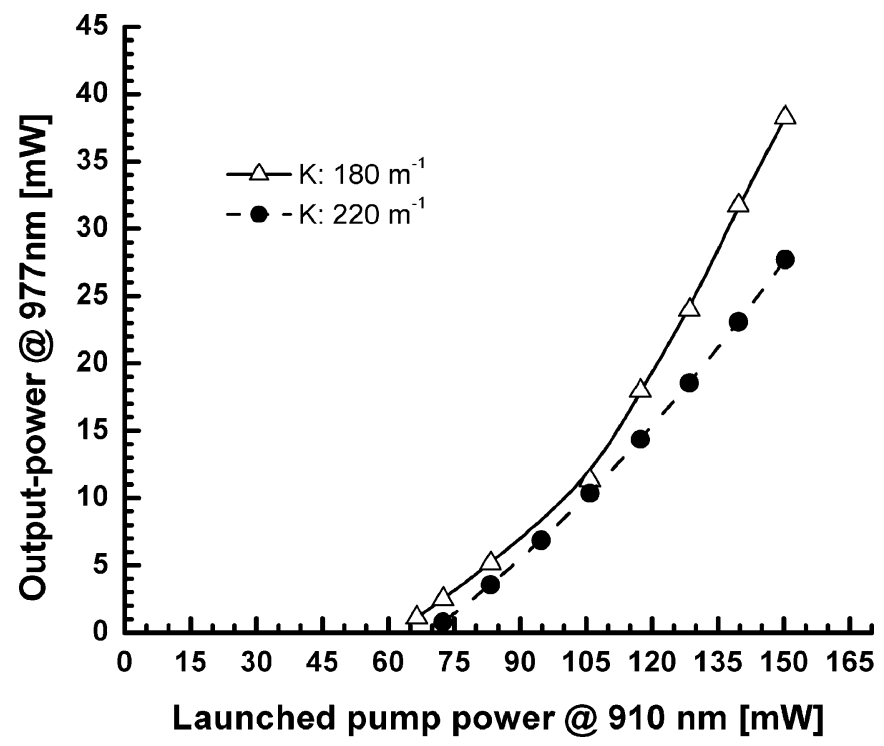

Fig. 2. Output power against pump power for different strength DFB gratings.

with slightly higher efficiency would be expected under 915-nm pumping because of a higher absorption at this wavelength as our investigations discussed below also reveal.

\section{EXPERIMENTAL RESULTS AND DISCUSSION}

Fig. 2 shows the output-power against launched pump power for two fiber DFB lasers of differing grating strength. We determine the grating strength using coupled mode theory in advance of the DFB laser inscription from short uniform gratings written in the same fiber. The solid line is for a DFB laser with a coupling strength $\kappa \sim 180 \mathrm{~m}^{-1}$ and with a threshold of about $60 \mathrm{~mW}$ of 910 -nm single-mode pump power. This laser provides an output power of $38.2 \mathrm{~mW}$ at Point E correcting for the splice and WDM insertion loss (the measured signal power from WDM Port $\mathrm{A}$ is $\sim 31 \mathrm{~mW}$ ) at a launched power of $150 \mathrm{~mW}$ at Point $\mathrm{E}$ (i.e., into the DFB fiber). The maximum slope efficiency is $61 \%$ with respect to launched pumping into the DFB fiber itself. The dashed line is for a DFB laser this time with a $\kappa \sim 220 \mathrm{~m}^{-1}$. This laser shows a slightly higher threshold power of $66 \mathrm{~mW}$ because of slightly higher splicing loss and slightly longer extra Er-Yb fiber before the DFB grating. For this laser, the maximum slope efficiency turns out to be substantially lower at just $37 \%$. We did not observe any mode hopping for both DFB lasers as the pump power varies from 60 to $150 \mathrm{~mW}$.

The relatively high thresholds powers, compared to more traditional wavelength fiber DFB lasers [1]-[8] occur because $>50 \% \mathrm{Yb}$-ion excitation is required to bleach the relatively high 977-nm reabsorption loss of this three-level laser transition. Together with this, the pump absorption at $910 \mathrm{~nm}$ is relatively low too. Additionally, note that for a given pump power of $113 \mathrm{~mW}$, the residual pump power after the 5.2-cm (1-mm additional length on either side of the DFB laser) $\mathrm{Yb}$ fiber is $\sim 29 \mathrm{~mW}$, measured at Port C. Furthermore, the penetration depth at $977 \mathrm{~nm}$ for a DFB grating with a designed coupling coefficient of $\kappa \sim 180 \mathrm{~m}^{-1}$ is $11 \mathrm{~mm}$ while for $\kappa \sim 220 \mathrm{~m}^{-1}$ it is only $9 \mathrm{~mm}$, thereby increasing the available gain and slope

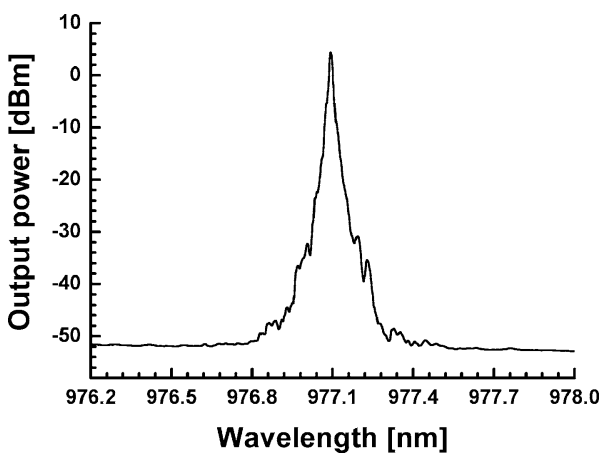

Fig. 3. Spectrum of the 977.1-nm fiber DFB laser, (resolution $0.01 \mathrm{~nm}$ ).

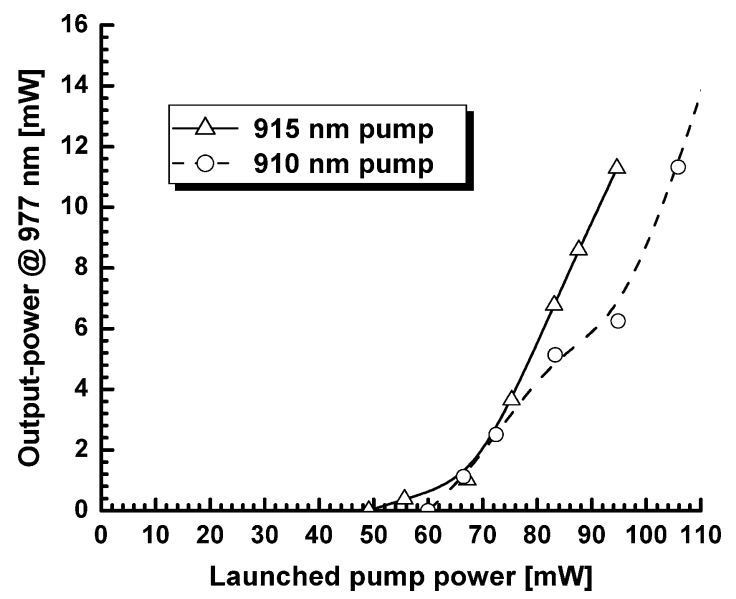

Fig. 4. Output power against pump power for different pump wavelengths, $\left(\kappa \sim 180 \mathrm{~m}^{-1}\right)$.

efficiency for the weaker grating. A typical power spectrum of the most efficient laser is shown in Fig. 3, it was recorded using an optical spectra analyzer with a resolution of $0.01 \mathrm{~nm}$ and clearly shows the operation of $977.1 \mathrm{~nm}$. Measurements using an optical spectrum analyzer indicate sidemode suppression ratio (SMSR) of at least $35 \mathrm{~dB}$ and an SNR of at least $55 \mathrm{~dB}$. Note that here the DFB with a $\kappa \sim 220 \mathrm{~m}^{-1}$ has slightly higher threshold due to a slightly higher splice loss at Point D. The polarization states were not investigated in our experiment. Supposing they are lasing in dual polarization states, it cannot be resolved fully using an optical spectrum analyzer with high resolution of $0.01 \mathrm{~nm}$ because the birefringence of this fiber and the birefringence introduced during UV writing is very small $\left(<5 \times 10^{-6}\right)$.

To investigate the possible higher efficiency of these fiber DFB lasers when pumped at $915 \mathrm{~nm}$, because of the better pump absorption, we used the output from a cladding-pumped dualclad Nd-doped fiber laser output as the pump source and used the same backward pump configuration as discussed above. We will report on the design and performance of this $\mathrm{Nd}$ fiber-laser elsewhere. The output power of the most efficient of the fiber DFB lasers $\left(\kappa \sim 180 \mathrm{~m}^{-1}\right)$ under both 910-nm laser diode and 915-nm fiber laser pump is plotted in Fig. 4. As demonstrated under 915-nm pumping, the threshold for the same fiber DFB laser is lowered by $\sim 15 \mathrm{~mW}$ which is believed mainly to be due to the higher pump absorption at this wavelength. Currently, we are limited in single-mode output power of the Nd-fiber laser to 
a relatively moderate $95 \mathrm{~mW}$, but these results do suggest that optimization of the pump wavelength within the 910-925-nm band for these 980-nm Yb-DFB lasers could enhance the total efficiency and that ultimately it should be possible to make extremely efficient single-frequency $980-\mathrm{nm}$ lasers by this route. Note the output power under the 910-nm pump is not so stable because this commercial pump laser diode is not cooled.

We believe that these low-noise fiber DFB lasers, for example, could present an ideal seed for high-power 980-nm lownoise amplifiers and could be very efficient for frequency-doubling applications because of their inherent narrow linewidth.

\section{CONCLUSION}

We have demonstrated efficient three-level operation of fiber DFB lasers at $977 \mathrm{~nm}$. The maximum slope efficiency against launched pump using a 910-nm single-mode pump diode of a 5-cm fiber DFB laser with coupling coefficient of $\kappa \sim 180 \mathrm{~m}^{-1}$ is demonstrated at $61 \%$. The laser has an SMSR of at least $35 \mathrm{~dB}$, an SNR of at least $55 \mathrm{~dB}$ and up to $38.2 \mathrm{~mW}$ of output power for a launched pump power of $150 \mathrm{~mW}$. We found that the use of a 915-nm single-mode fiber laser as the pump source could lower the threshold and potentially further enhance the total efficiency despite the fact that we are, at present, limited in total power at this wavelength.

\section{ACKNOWLEDGMENT}

The authors wish to acknowledge Prof. A. B. Grudinin from Fianium Ltd. for the loan of the 910-nm single-mode pump diode.

\section{REFERENCES}

[1] M. Ibsen, S. Y. Set, C. S. Goh, and K. Kikuchi, "Broad-band continuously tunable all-fiber DFB lasers," IEEE Photon. Technol. Lett., vol. 14, pp. 21-23, Jan. 2002.

[2] J. T. Kringlebotn, J. L. Archambault, L. Reekie, and D. N. Payne, " $\mathrm{Er}^{3+}: \mathrm{Yb}^{3+}$ co-doped fiber distributed-feedback laser," Opt. Lett., vol. 19, pp. 2101-2103, 1994.

[3] M. Ibsen, E. Rønnekleiv, G. J. Cowle, M. O. Berendt, O. Hadeler, M. N. Zervas, and R. I. Laming, "Robust high power $(>20 \mathrm{~mW})$ all-fiber DFB lasers with unidirectional and truly single polarization outputs," in Proc. CLEO '99, Baltimore, MD, 1999, Paper CWE4.

[4] L. B. Fu, M. Ibsen, P. W. Turner, D. J. Richardson, and D. N. Payne, "Keyed-axis single-polarization all-fiber DFB laser," Electron. Lett., vol. 38, no. 24, pp. 1537-1539, 2002.

[5] P. Varming, V. Lauridsen, J. H. Povlsen, J. B. Jensen, M. Kristensen, and B. Palsdottir, "Design and fabrication of Bragg grating based DFB fiber lasers operating above $1610 \mathrm{~nm}$," in Proc. Optical Fiber Communication (OFC 2000), vol. 3, Baltimore, MD, 2000, Paper ThA6, pp. 17-19.

[6] L. B. Fu, M. Ibsen, D. J. Richardson, and D. N. Payne, "Highly efficient $L$-band fiber-DFB lasers," in Proc. Eur. Conf. Optical Communication (ECOC 2003), vol. 3, Rimini, Italy, 2003, Paper We2.6.6, pp. 472-473.

[7] A. Asseh, H. Storøy, J. T. Kringlebotn, W. Margulis, B. Sahlgren, S. Sandgren, R. Stubbe, and G. Edwall, " $10 \mathrm{~cm} \mathrm{Yb}^{3+}$ DFB fiber laser with permanent phase shifted grating," Electron. Lett., vol. 31, no. 12, pp. 969-970, 1995.

[8] R. Selvas, K. Yla-Jarkko, J. K. Sahu, L. B. Fu, J. N. Jang, J. Nilsson, S.-A. Alam, P. W. Turner, J. Moore, and A. B. Grudinin, "High power, low noise Yb-ring-doped cladding-pumped three-level fiber laser," Opt. Lett., vol. 28, no. 13, pp. 1093-1095, 2003.

[9] L. Dong, W. H. Loh, J. E. Caplen, J. D. Minelly, K. Hsu, and L. Reekie, "Efficient single-frequency fiber lasers using novel photosensitive $\mathrm{Er} / \mathrm{Yb}$ optical fibers," Opt. Lett., vol. 22, no. 13, pp. 694-96, 2003. 